\title{
Non-Linear Electronic Effects in Cluster-Solid Interactions
}

\author{
E.Parilis \\ California Institute of Technology , 200-36, Pasadena, CA , 91125. \\ A review will be given of the effects of non- additivity under impacts of clusters, observed only in a variety \\ of electronic energy loss phenomena. They include electron emission, luminescence, projectile charge state formation, electron- \\ hole pairs generation causing the surface barrier detector response and the high-energy part of the secondary ion emission under \\ cluster impacts. The sublinear effects consist in decreasing the yields per one incident atom with increasing number of cluster \\ constituents at the same velocity. The ratio of the yields $R_{n}=Y_{n} / n Y_{1}$, being the measure of the effect, is less than 1 and \\ usually decreases with increasing projectile velocity, and the number of atoms in the cluster $n$.
}

The reduction is caused by the mechanism of sweeping - out - electrons, that removes some electrons from the cluster trek in binary collisions between the front running cluster atoms and the target atoms, leaving fewer available for the tailing ones.

\section{INTRODUCTION}

After decades of studying the secondary emission under ion bombardment, the time had came to investigate the same processes under impacts of molecules and clusters. The first question was: are all the effects additive? Is there a dependence of the secondary yields per atom on the number of atoms in a cluster impinging the solid surface at the same velocity?

Along with the linear phenomena, a group of nonlinear effects has been discovered. They consist in an enhancement, in some cases very drastic, of the yield per atom, with increasing number of atoms in the cluster. All of them are connected to the nuclear stopping and could be regarded as a natural result of the synergetic action of the cluster constituents.

What was puzzling and intriguing and yet not completely explained was the existence of some non additivity effects, in which the total yield is sublinear, i.e. it is less than proportional to the number of atoms in the cluster. The extent of reduction increased with the increasing number of cluster constituents. It looked like the atoms were mutually blocking or screening each other, diminishing the resulting effect. All the sublinear effects were observed only in the electronic stopping related phenomena. They were discovered in different laboratories, studying the secondary electron emission, luminescence, and projectile charge state formation after passing through thin films. Clusters and polyatomic molecules in wide range of energies, from a few $\mathrm{KeV} /$ atom to $4 \mathrm{MeV} /$ atom, were used as projectiles. It was supposed that the sublinear reduction is due to a sweeping - out - electrons effect, consisting in removing some electrons from the cluster trek in binary collisions between the front running cluster atoms and target atoms, leaving fewer available for the tailing ones.

\section{SECONDARY ELECTRON EMISSION}

A sublinear effect in the secondary electron emission from CsI under impacts of gold clusters $\mathrm{Au}_{\mathrm{n}}$ has been observed experimentally by the group of $\mathrm{Y}$. Le Beyec at IPN, Orsay. The effect manifests itself in a decrease of the electron yield per one cluster atom $\gamma_{\mathrm{n}}(\mathrm{E}) / \mathrm{n}$ with increasing number of atoms in the cluster $n$. The ratio $R_{n}=\gamma_{n}(E) / n \gamma_{1}(E)$, being a measure of the effect, is less than 1 and decreased with 
$\mathrm{n}$ and the projectile energy $\mathrm{E}$ The mechanism of sweeping-out-electrons was proposed to explain this effect [1]. It supposes that the number of available electrons after passage of each cluster atom decreases in a geometrical progression with a factor $\left(1-\sigma_{\mathrm{e}} / \pi \mathrm{r}_{\mathrm{n}}{ }^{2}\right)$. That gives

$\mathrm{R}_{\mathrm{n}}=\gamma_{\mathrm{n}} / \mathrm{n} \gamma_{1}=\left[1-\left(1-\sigma_{\mathrm{e}} / \pi \mathrm{r}_{\mathrm{n}}^{2}\right)^{\mathrm{n}}\right] /\left(\mathrm{n} \sigma_{\mathrm{e}} / \pi \mathrm{r}_{\mathrm{n}}^{2}\right)$

the ratio $\sigma_{\mathrm{e}} / \pi \mathrm{r}_{\mathrm{n}}{ }^{2}$ could be replaced by $\delta_{\mathrm{n}}(\mathrm{dE} / \mathrm{dx})_{\mathrm{e}}$ where $\sigma_{\mathrm{e}}$ is ionization cross-section, $\mathrm{r}_{\mathrm{n}}$ are the cluster radii, $\rho$ is the atomic density, $\delta_{n}=k_{e} / \rho \pi r_{n}^{2}$ and $k_{e}$ ( ions/eV ) is the efficiency of the electronic energy losses $(\mathrm{dE} / \mathrm{dx})_{\mathrm{e}}$ for internal ionization. The dependence $R_{n}(E)$ is determined by the trend of $(d E / d x)_{e}$ which usually increases with E.

A similar nonlinear effect in electron emission from carbon films under hydrogen cluster impacts has been observed in Lyon [2] .Y.Susuki [3] found the vicinage effect in electron emission from SnTe surface induced by hydrogen clusters $\mathrm{H}_{2}$ and $\mathrm{H}_{3}$. The values of $R_{n}$ and their energy dependence were in a good agreement with a calculation using the above equation. S.M.Ritzau and R.A.Baragiola [4] found a molecular effect in secondary electron emission in the backward direction from carbon foils under 0.5-50 $\mathrm{keV}$ /a.m.u. $\mathrm{H}_{2}, \mathrm{CO}, \mathrm{N}_{2}$ and $\mathrm{O}_{2}$ ion bombardment. In a study by E.Veje [5] a similar effect has been observed for electron emission induced by $\mathrm{HeH}$, which is compared to the sum of electron yields for $\mathrm{H}$ and $\mathrm{He}$ at the same velocity. Recently A.M. Borisov and E.S.Mashkova [6] observed a molecular effect in the electron emission from graphite, chromium and gold under $\mathrm{N}_{2}$ with the energy $15-34 \mathrm{KeV}$.

A sublinear effect in kinetic electron emission from CsI under impacts of very slow ( the velocity $\mathrm{v}=5 \times$ $10^{5}-7 \times 10^{6} \mathrm{~cm} / \mathrm{s}$ ) and large (the mass $M=1182$ 66430 a.m.u.) biomolecules has been reported by the Orsay group [7] Given that the bioorganic molecules have very close elemental composition, one can take the mass of the biomolecule LHRH (M=1182 a.m.u.) as unity, $\mathrm{n}=1$. Then for INSULIN (M=5733 a.m.u.) $\mathrm{n}$ $\approx 5$, for TRYPSIN (M=23296 a.m.u.) $\mathrm{n} \approx 20$ and for ALBUMIN (M=66430 a.m.u.) n $\approx 56$, approximately.

One can calculate from the experimental data the ratios $R_{n}=\gamma_{n} / n \gamma_{1}$ with $\gamma_{1}$ being the electron emission yield for LHRH . They were equal to 0.25 0.75 and decreased with both $\mathrm{n}$ and $\mathrm{v}$ increasing in accordance with the equation (1).

\section{LUMINESCENCE UNDER MOLECULAR ION BOMBARDMENT}

One should expect a similar sublinear effect in light emission from solids under cluster impacts. Indeed E.Schweikert's group of Texas A\&M University observed a reduction, with $\mathrm{n}$ and $\mathrm{E}$ increasing, in the number of photons per atom emitted from CsI bombarded with polyatomic projectiles $\mathrm{H}_{\mathrm{n}}^{+},(\mathrm{NaF})_{\mathrm{n}}$ $\mathrm{N}^{+}(\mathrm{n}=1,2), \mathrm{Na}^{+}, \mathrm{Cs}_{2} \mathrm{I}^{+}$, and $\mathrm{Cs}^{+}$at impact energy $\mathrm{E}=5-30 \mathrm{keV}$. The effect has been attributed to the mechanism of sweeping out electrons, and the same formalism was applied to calculate it [8]. The formula for the reduction factor $\mathrm{R}_{\mathrm{nm}}$, has been generalized for heteroatomic clusters containing $\mathrm{n}$ atoms of type a and $\mathrm{m}$ atoms of type $\mathrm{b}$ :

$\mathrm{R}_{\mathrm{nm}}(\mathrm{v}, \mathrm{x})=\left[1-\left(1-\delta_{\mathrm{nm}}(\mathrm{dE} / \mathrm{dx})_{\mathrm{a}}\right)^{\mathrm{n}}\left(1-\delta_{\mathrm{nm}}(\mathrm{dE} / \mathrm{dx})_{\mathrm{b}}\right)^{\mathrm{m}}\right]$

$/\left[\mathrm{n} \delta_{\mathrm{nm}}(\mathrm{dE} / \mathrm{dx})_{\mathrm{a}}+\mathrm{m} \delta_{\mathrm{nm}}(\mathrm{dE} / \mathrm{dx})_{\mathrm{b}}\right]$,

In the same year Koch, Tuszynski, Tomaschko, and Voit [9] have studied the photon emission under 1 $\mathrm{MeV}$ carbon cluster $\mathrm{C}_{\mathrm{n}}(\mathrm{n}=2-10)$ impacts. They found that the luminescence exhibits a very strong sublinear effect ranging from $R_{n}=0.9-0.6$ for CsI to $0.5-0.2$ for POPOP.

\section{CHARGE STATE OF CLUSTER ATOMS PASSING THROUGH THIN FOILS}

The most recent manifestation of the sweeping-outelectrons mechanism was found by the same group of IPN, Orsay, having studied the charge state of fast atoms passing through solids [10]. They have observed the mean charge state $\mathrm{q}_{\mathrm{n}}$ of carbon atoms, passing through a thin carbon foil $(\mathrm{s}=100-2000 \AA)$ with energy 1,2 and $4 \mathrm{MeV} /$ at, in clusters $C_{n}(n=3,5,8$ and 10), being smaller than the mean charge $\mathrm{q}_{1}$ of a single carbon atom $\mathrm{C}_{1}$ passing through the same foils with the same velocity. The curves $R_{n}(s)<1$, show an explicit decrease with $\mathrm{n}$ and increase with the increasing path in the solid $\mathrm{s}$, approaching $\mathrm{R}_{\mathrm{n}}=1$ at large $s$, or a decrease with the exit energy $E$.

Each of the front running cluster atoms, forming its charge in binary collision with target atoms, increases the ionization energy of the cluster, which equals $\mathrm{V}_{\mathrm{n}}=\mathrm{V}+\mathrm{n} \mathrm{q}_{\mathrm{n}} \mathrm{e}^{2} / \mathrm{r}_{\mathrm{n}}$, by a factor

$$
1+\sigma(s) / \pi r_{n}^{2}=1+\delta_{n}(d E / d x)_{e},
$$


where $\delta_{n}=\left(\pi r_{n}^{2} J \rho\right)^{-1}$ and $V$ is the ionization energy of one atom. In the mean time the number of target atoms available for stripping collisions with the same cross section is reduced by $1-\delta_{\mathrm{n}}(\mathrm{dE} / \mathrm{dx})_{\mathrm{e}}$. The total charge $\mathrm{Q}_{\mathrm{n}}$ is a sum of a geometrical progression with the factor $\left(1-\delta_{\mathrm{n}}(\mathrm{dE} / \mathrm{dx})_{\mathrm{e}}\right) /\left(1+\delta_{\mathrm{n}}(\mathrm{dE} / \mathrm{dx})_{\mathrm{e}}\right)$, and the ratio $R_{n}$ equals

$$
\begin{aligned}
& \mathrm{R}_{\mathrm{n}}=\mathrm{q}_{\mathrm{n}} / \mathrm{q}_{1}=\mathrm{Q}_{\mathrm{n}} /\left(\mathrm{n} \mathrm{q}_{1}\right)= \\
& \left\{1-\left[\left(1-\delta_{\mathrm{n}}(\mathrm{dE} / \mathrm{dx})_{\mathrm{e}}\right) /\left(1+\delta_{\mathrm{n}}(\mathrm{dE} / \mathrm{dx})_{\mathrm{e}}\right)\right]\right\} / \\
& \left\{2 \mathrm{n} \delta_{\mathrm{n}}(\mathrm{dE} / \mathrm{dx})_{\mathrm{e}} /\left(1+\delta_{\mathrm{n}}(\mathrm{dE} / \mathrm{dx})_{\mathrm{e}}\right)\right\},
\end{aligned}
$$

The equation (3) gives an increase of $R_{n}$ with increasing path $\mathrm{s}$, caused both by decreasing $(\mathrm{dE} / \mathrm{dx})_{\mathrm{e}}$ and by increasing separation of the cluster atoms $r_{n}$ due to Coulomb repulsion.

The calculated curves $R_{n}(s)[11,12]$ were compared to the experimental ones [10]. It was found, that the sequence of the curves, their mutual distance and the general trend with s agree well with the experiment.

\section{ELECTRONIC SPUTTERING}

The process of sweeping out electrons should affect also the electronic sputtering under high-energy cluster impacts. The number of ionized atoms $\mathrm{N}_{\mathrm{i}}$ left after each consecutive cluster atom has passed the charged trek cylinder is diminished by a factor $1-\sigma_{\mathrm{i}} / \pi \mathrm{r}_{\mathrm{n}}{ }^{2}$, where $\sigma_{i}$ is the ionization cross-section and $r_{n}$ is the cluster radius. The corresponding ratio $R_{n}=Y_{n} / n Y_{1}$ is $<\mathbf{1}$ and should decrease with the projectile energy per atom $\mathrm{E}$ and the number of cluster constituents $\mathrm{n}$. To describe the non-linearity one uses usually the ratios

$$
\begin{aligned}
& \varepsilon^{\mathrm{n}}{ }_{\mathrm{m}}=m Y_{\mathrm{n}} / \mathrm{nY}_{\mathrm{m}}=\mathrm{R}_{\mathrm{n}} / \mathrm{R}_{\mathrm{m}} \quad(\mathrm{n}>\mathrm{m}) \quad \text { or } \\
& \varepsilon^{\mathrm{n}}{ }_{\mathrm{n}-1}=(\mathrm{n}-1) \mathrm{Y}_{\mathrm{n}} / \mathrm{nY}_{\mathrm{n}-1}=\mathrm{R}_{\mathrm{n}} / \mathrm{R}_{\mathrm{n}-1} \quad(\mathrm{~m}=\mathrm{n}-1)
\end{aligned}
$$

According to the above, $\varepsilon^{\mathrm{n}}{ }_{\mathrm{n}-1}<1$ and decreases with $\mathrm{E}$, but increases with $\mathrm{n}$.

Indeed, in the experiments on secondary ion emission by $10^{2}-10^{4} \mathrm{KeV} / \mathrm{at} \mathrm{Au}_{\mathrm{n}}$ clusters [13] it was observed, that the ratios $\varepsilon^{n}{ }_{n-1} \quad(n=2-4)$ sharply decrease with $\mathrm{E}$ and could be projected both to cross the line $\varepsilon{ }^{n}-1=1$ and to change to the opposite the sequence of the curves around $\mathrm{E}>4 \mathrm{MeV} /$ at (see
Fig.11 of Ref.[13]), where the electronic energy losses exceed the nuclear ones, albeit the yield still cannot be attributed fully to the electronic sputtering. The calculation predicts such a trend for electronic sputtering.This could be possibly verified by extension the experiments further beyond $\mathrm{E}>4 \mathrm{MeV} / \mathrm{at}$.

The experiment shows a linear increase of $Y_{n}$ with $n$ in contrast to the $Y_{n} \propto n^{3}$ or $Y_{n} \propto n^{2}$ enhancement at lower projectile energies, where electronic sputtering is inferior to the nuclear one. The deviation is caused by the sweeping-out-electrons effect which predicts for electronic sputtering $\mathrm{Yn} \propto \mathrm{n}^{\mathrm{b}}$ with $\mathrm{b}<1$. Around $\mathrm{E}=4 \mathrm{MeV} / \mathrm{at}, \mathrm{b}=0.6$, while for electron

emission yield $\gamma$ under $\mathrm{Au}_{\mathrm{n}}$ [1], which is linear with $(\mathrm{dE} / \mathrm{dx})_{\mathrm{e}}, \mathrm{b}=0.26$. The final trend of $\mathrm{Y}_{\mathrm{n}}(\mathrm{n})$ depends on the shares of electronic and nuclear sputtering in the total yield.

\section{SURFACE BARRIER DETECTOR PULSE HEIGHT DEFECT}

- The same mechanism could explain the pulse height defect (PHD) in a surface barrier detector under $\mathrm{MeV}$ cluster impacts [14]. While other models of PHD deal just with e-h annihilation, the sweeping-outelectrons model explains its very existence by the reduction in the electron-hole pair creation in binary collisions.. A recent estimate of the contribution into $\Delta_{\mathrm{r}}$ caused by sweeping-out-electrons effect gives a correct dependence on both $\mathrm{n}$ and $\mathrm{E}$ (Fig.1). The dependence $\Delta_{\mathrm{r}} \propto \mathrm{n}(\mathrm{n}-1)$ comes naturally from this mechanism, while $\Delta_{\mathrm{r}} \propto \mathrm{n}^{2}$ makes no sense and, by definition, cannot be used even to discuss the experimental data.

\section{CONCLUSION}

What other phenomena should we expect to be affected by the mechanism of sweeping - out electrons? Probably everything that is caused by the electronic stopping: creation of radiation defects, color centers, induced conductivity, desorption of large biomolecules, emission of multiply charged ions and $\mathrm{X}$-rays.The main goal of this paper was to attract attention to a peculiar and yet neither well studied, nor fully understood effect, which has been observed in too many phenomena to be regarded as just an isolated event. 
PHD Under $\mathrm{C}_{\mathrm{n}}$ Impacts on $\mathrm{Si}$ Detector

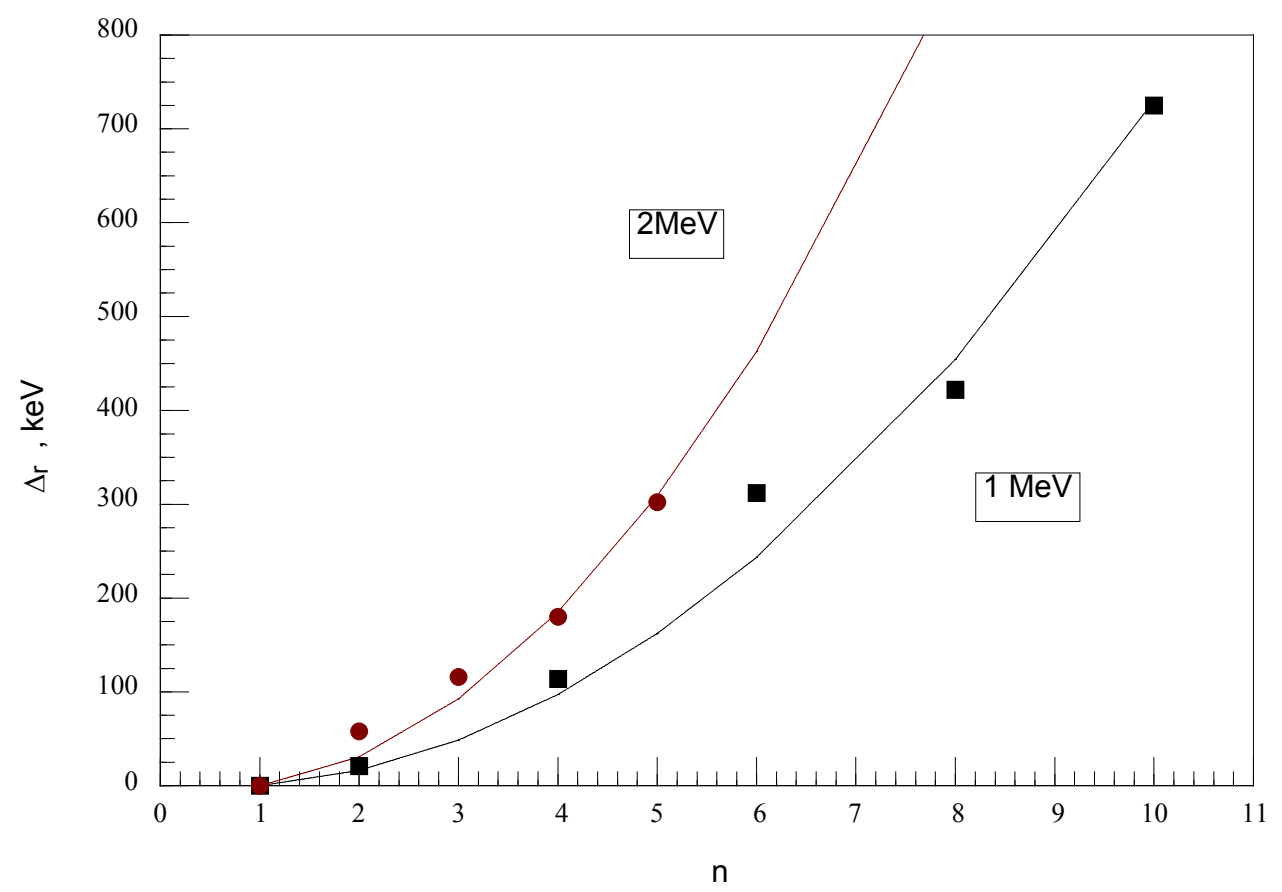

FIGURE 1. A most recent example of the sweepingout-electrons effect: the PHD under $\mathrm{C}_{\mathrm{n}}$ impacts on $\mathrm{Si}$ detector, $\Delta_{\mathrm{r}}$, depends on $\mathrm{n}$ as $\mathrm{n}(\mathrm{n}-1)$ and increases with the energy $\mathrm{E}$ increasing from 1 to $2 \mathrm{MeV} /$ at.

\section{REFERENCES}

1. K. Baudin, A.Brunelle, S.Della-Negra, J.Depauw, Y. Le Beyec and E.Parilis, Nucl.Instrum. \& Methods B 117, 47 (1996).

2.A.Billebaud,D.Dauvergne,M.Fallavier,R.Kirsh,J.-C.Poizat, J.Remillieux, J.-P.Tomas and H.Rothard Nucl. Instrum. Methods B 112,79 (1996); Radiation Effects and Defects in Solids, 126, 373 (1993). M. Fallavier, R. Kirsch, J.C. Poizat, J. Remillieux and J.P. Thomas, Nucl. Instr. \& Meth. in Phys. Res. B164 -165 (2000) 920

3. Y.Susuki, Z. Phys. D, 42,293 (1997).

4. S.M.Ritzau and R.A.Baragiola, Phys.Rev. B 58, 2529 (1998).

\section{E. Veje, Nucl. Instrum. Methods 194 (1982) 433.}

6. A.M.Borisov, E.S.Mashkova, E.S.Parilis,Vacuum, 66, 145-148 (2002)
7.A.Brunelle,P.Chaurand, S.Della-egra,Y.LeBeyec,E.Parilis, Rapid Commun. Mass Spectrom., 11, 353 (1997).

8. K.Baudin, E.S.Parilis, J.F.Blankenship, M.J.Van Stipdonk, E.A.Schweikert, Nucl. Instrum. Methods B 134 ,352 (1998).

9. K. Koch, W. Tuszynski, Ch. Tomaschko, H.Voit, Nucl. Instrum. Methods B 146, 198 (1998)

10. A. Brunelle, S.Della-Negra, J. Depauw,D. Jacquet,Y.Le Beyec, M. Pautrat, Phys.Rev., A 59_, 4456 (1999).

11. E.Parilis, Charge State of Carbon Atoms Passing Through Thin Foils, Report to IPN, Orsay, 1998

12 E.Parilis, Nuclear Instruments and Methods in Physics Research B,Vol. 193 (1-4) (2002) pp. 240-247

13.A. Brunelle, S.Della-Negra, J. Depauw,D. Jacquet, Y.Le Beyec, M. Pautrat, K.Baudin, H.H. Andersen , Phys.Rev., A 63, 022902 (2001).

14. M. Seidl, H.Voit, S. Bouneau, A. Brunelle, S. DellaNegra, J. Depauw, D. Jacquet, Y.Le Beyec, M. Pautrat, Nucl. Instr. Methods Phys. Res B 183 (2001) 502-508 